\title{
Rational and real positive semidefinite rank can be different
}

\author{
Hamza Fawzi* \\ Laboratory for Information and Decision Systems (LIDS) \\ Massachusetts Institute of Technology, Cambridge, MA 02139, USA \\ João Gouveia \\ CMUC, Department of Mathematics, University of Coimbra, 3001-454 Coimbra, Portugal \\ Richard Z. Robinson \\ Department of Mathematics, University of Washington, Box 354350, Seattle, WA 98195, USA
}

\begin{abstract}
Given a $p \times q$ nonnegative matrix $M$, the psd rank of $M$ is the smallest integer $k$ such that there exist $k \times k$ real symmetric positive semidefinite matrices $A_{1}, \ldots, A_{p}$ and $B_{1}, \ldots, B_{q}$ such that $M_{i j}=\left\langle A_{i}, B_{j}\right\rangle$ for $i=1, \ldots, p$ and $j=1, \ldots, q$. When the entries of $M$ are rational it is natural to consider the rationalrestricted psd rank of $M$, where the factors $A_{i}$ and $B_{j}$ are required to have rational entries. It is clear that the rational-restricted psd rank is always an upper bound to the usual psd rank. We show that this inequality may be strict by exhibiting a matrix with psd rank four whose rational-restricted psd rank is strictly greater than four.
\end{abstract}

Keywords: Matrix factorization, Positive semidefinite rank, Semidefinite programming 2010 MSC: 15A23, 90C22

Given a $p \times q$ nonnegative matrix $M$, the positive semidefinite (psd) rank of $M$, denoted $\operatorname{rank}_{\mathrm{psd}} M$, is the smallest integer $k$ such that there exist $k \times k$ real symmetric positive semidefinite matrices $A_{1}, \ldots, A_{p}$ and $B_{1}, \ldots, B_{q}$ such that $M_{i j}=\left\langle A_{i}, B_{j}\right\rangle$ for $i=1, \ldots, p$ and $j=1, \ldots, q$. Any such collection of matrices $A_{i}$ and $B_{j}$ is called a psd factorization of $M$. The notion of psd rank was introduced in [1] (see also [2]) and has many appealing geometric interpretations, including semidefinite representations of polyhedra and information-theoretic applications. We refer to [3] for a survey on this notion and a review of recent literature on it.

In this note, we answer a basic structural question about the psd rank: if a nonnegative matrix $M$ has only rational entries, can the psd rank of $M$ always be achieved by a factorization using only rational matrices? We answer this question negatively by providing an example of a rational matrix with psd rank four such that every psd factorization of size four uses irrational numbers. Note that the analogous question for the nonnegative rank of a matrix was posed by Cohen and Rothblum in [4]. It was shown in [4] that all rational matrices with nonnegative rank two admit a rational nonnegative factorization, but the question for general nonnegative matrices remains open. The recent paper [5] however shows that there is a subfield $F$ of $\mathbb{R}$ and a matrix $A$ with entries from $F$ such that the nonnegative rank of $A$ over $F$ is strictly greater than the nonnegative rank of $A$ over $\mathbb{R}$.

The proof of our example will require a lemma about rational psd matrices of rank one. Any rank one symmetric psd matrix has the form $\mathbf{v v}^{T}$ for some vector $\mathbf{v}$. Then we have the following.

Lemma 1. If the matrix $\mathbf{v} \mathbf{v}^{T}$ is composed of only rational entries, then $\mathbf{v}$ has the form $\alpha \mathbf{q}$ where $\alpha$ is a real scalar and $\mathbf{q}$ is a rational vector.

Proof. Suppose that $\mathbf{v}$ is a nonzero vector (else the conclusion is immediate). Then, without loss of generality, we may assume that the first coordinate $v_{1}$ is nonzero. Since $v_{1}^{2}$ is an entry in the matrix $\mathbf{v} \mathbf{v}^{T}$, it must be

*Corresponding author. Email: hfawzi@mit.edu.

Preprint submitted to Elsevier

November 20, 2015

(C) 2015. This manuscript version is made available under the Elsevier user license

http://www.elsevier.com/open-access/userlicense/1.0/ 


$$
M=\left(\begin{array}{llllll}
0 & 0 & 2 & 1 & 0 & 1 \\
1 & 0 & 0 & 2 & 0 & 1 \\
0 & 1 & 2 & 0 & 0 & 1 \\
1 & 2 & 0 & 0 & 0 & 1 \\
0 & 0 & 2 & 1 & 1 & 0 \\
1 & 0 & 0 & 2 & 1 & 0 \\
0 & 1 & 2 & 0 & 1 & 0 \\
1 & 2 & 0 & 0 & 1 & 0
\end{array}\right)
$$

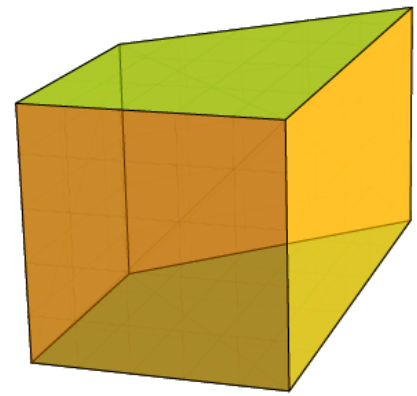

Figure 1. Our example matrix is a slack matrix of a three dimensional polytope.

rational. Hence, the matrix $\frac{1}{v_{1}^{2}} \mathbf{v} \mathbf{v}^{T}$ is also rational. By looking at the first row of this matrix, we see that the vector $\left(1, \frac{v_{2}}{v_{1}}, \frac{v_{3}}{v_{1}}, \ldots, \frac{v_{r}}{v_{1}}\right)$ is rational. Now we just scale this rational vector by $v_{1}$ to finish the proof.

Our candidate matrix $M$ is the $8 \times 6$ matrix shown in Figure 1 . Readers who are familiar with slack matrices may be interested to know that $M$ arises as a slack matrix of the polytope with vertices $(0,0,0)$, $(1,0,0),(0,1,0),(1,2,0),(0,0,1),(1,0,1),(0,1,1)$, and $(1,2,1)$. Readers who are not familiar with slack matrices need not worry, as we will refrain from using any results about slack matrices in the proofs.

During our analysis of this example, we will require a few results about psd rank found in the literature. A matrix $S$ is called an entry-wise square root of $A \in \mathbb{R}_{+}^{p \times q}$ if $S_{i j}^{2}=A_{i j}$ for all $i=1, \ldots, p$ and $j=1, \ldots, q$. We summarize these results in the following proposition.

\section{Proposition 2.}

1. [1, Prop. 5] If $S$ is an entry-wise square root of $A$, then $\operatorname{rank}_{\mathrm{psd}} A \leq \operatorname{rank} S$.

2. [6, Cor. 4.8], [7, Prop. 2.6] If A contains a $k \times k$ triangular submatrix T, then $\operatorname{rank}_{\mathrm{psd}} A \geq k$. Furthermore, in a psd factorization of $A$ of size $k$, the factor corresponding to the row (or column) of $T$ with $k-1$ zeros must have rank one.

Now we begin our analysis of the matrix $M$.

Lemma 3. We have that $\operatorname{rank}_{\mathrm{psd}} M=4$ and any psd factorization of $M$ of size four uses only rank one factors.

Proof. One can verify that the entry-wise square root of $M$ with all entries nonnegative has usual rank four. Thus Proposition 2 says that $\operatorname{rank}_{\mathrm{psd}} M \leq 4$. Consider the submatrix of $M$ indexed by rows $1,5,7$, and 8 and columns 1, 2, 5, and 6. This submatrix is triangular so Proposition 2 tells us two things: First, we have that $\operatorname{rank}_{\mathrm{psd}} M \geq 4$ and, hence, $\operatorname{rank}_{\mathrm{psd}} M=4$. Second, the factors corresponding to the first row and first column in a psd factorization of $M$ of size four must always be rank one. It is easy to verify by inspection that for every row and column of $M$ we can find a $4 \times 4$ triangular submatrix such that the row or column in question has three zeros in that submatrix. Thus, repeatedly applying the proposition completes the proof.

Remark 4. Note that Lemma 3 is actually a consequence of [7, Prop. 3.2] since our polytope has minimal psd rank (equal to the ambient dimension plus one) and thus any psd factorization must consist entirely of rank-one factors.

The next proposition shows that no rational psd factorization of $M$ can have size four.

Proposition 5. We have that $\operatorname{rank}_{\mathrm{psd}} M=4$, but there does not exist a psd factorization of size four using only rational matrices.

Proof. Suppose, by way of contradiction, that $\left(A_{1}, \ldots, A_{8}, B_{1}, \ldots, B_{6}\right)$ is a psd factorization of $M$ of size four that uses only rational matrices. By Lemma 3, each matrix must be rank one. Thus, there exist vectors $\mathbf{a}_{\mathbf{1}}, \ldots, \mathbf{a}_{\mathbf{8}}$ and $\mathbf{b}_{\mathbf{1}}, \ldots, \mathbf{b}_{\mathbf{6}}$ such that $A_{i}=\mathbf{a}_{\mathbf{i}} \mathbf{a}_{\mathbf{i}}{ }^{T}$ and $B_{j}=\mathbf{b}_{\mathbf{j}} \mathbf{b}_{\mathbf{j}}{ }^{T}$. Furthermore, by the properties of the trace, 
we must have that $M_{i j}=\left\langle A_{i}, B_{j}\right\rangle=\left\langle\mathbf{a}_{\mathbf{i}}, \mathbf{b}_{\mathbf{j}}\right\rangle^{2}$. Thus, the matrix whose $(i, j)$ th entry is given by $\left\langle\mathbf{a}_{\mathbf{i}}, \mathbf{b}_{\mathbf{j}}\right\rangle$ is an entry-wise square root of $M$, which we denote by $S$. By looking at the submatrix of $S$ corresponding to the first two rows and the fourth and sixth columns, we see that $S$ contains a submatrix $\widetilde{S}$ of the form

$$
\left(\begin{array}{cc} 
\pm 1 & \pm 1 \\
\pm \sqrt{2} & \pm 1
\end{array}\right)
$$

where there is ambiguity on the sign of each entry.

Now by Lemma 1 , each $\mathbf{a}_{\mathbf{i}}$ and $\mathbf{b}_{\mathbf{j}}$ must be a rational vector scaled by a nonzero real number. Hence, there must exist nonzero real numbers $\alpha_{1}, \alpha_{2}, \beta_{1}, \beta_{2}$ such that the matrix resulting from the product

$$
\left(\begin{array}{cc}
\alpha_{1} & 0 \\
0 & \alpha_{2}
\end{array}\right) \cdot \widetilde{S} \cdot\left(\begin{array}{cc}
\beta_{1} & 0 \\
0 & \beta_{2}
\end{array}\right)=\left(\begin{array}{cc} 
\pm \alpha_{1} \beta_{1} & \pm \alpha_{1} \beta_{2} \\
\pm \sqrt{2} \alpha_{2} \beta_{1} & \pm \alpha_{2} \beta_{2}
\end{array}\right)
$$

is rational. But if $\alpha_{1} \beta_{1}, \alpha_{1} \beta_{2}$, and $\alpha_{2} \beta_{2}$ are rational, then $\alpha_{2} \beta_{1}$ must also be rational which means that $\pm \sqrt{2} \alpha_{2} \beta_{1}$ is irrational and this is a contradiction.

\section{Acknowledgements}

Fawzi was supported in part by AFOSR FA9550-11-1-0305. Gouveia was supported by the Centre for Mathematics at the University of Coimbra and Fundação para a Ciência e a Tecnologia, through the European program COMPETE/FEDER and Robinson was supported by the US NSF Graduate Research Fellowship through grant DGE-1256082.

\section{References}

[1] J. Gouveia, P. Parrilo, R. Thomas, Lifts of convex sets and cone factorizations, Mathematics of Operations Research 38 (2) (2013) 248-264.

[2] S. Fiorini, S. Massar, S. Pokutta, H. Tiwary, R. de Wolf, Linear vs. semidefinite extended formulations: Exponential separation and strong lower bounds, in: Proceedings of the Forty-fourth Annual ACM Symposium on Theory of Computing, STOC '12, ACM, 2012, pp. 95-106.

[3] H. Fawzi, J. Gouveia, P. A. Parrilo, R. Z. Robinson, R. R. Thomas, Positive semidefinite rank, Mathematical Programming 153 (1) (2015) 133-177.

[4] J. Cohen, U. Rothblum, Nonnegative ranks, decompositions, and factorizations of nonnegative matrices, Linear Algebra and its Applications 190 (1993) 149-168.

[5] Y. Shitov, Nonnegative rank depends on the field, arXiv preprint arXiv:1505.01893.

[6] T. Lee, D. O. Theis, Support-based lower bounds for the positive semidefinite rank of a nonnegative matrix, arXiv preprint arXiv:1203.3961v4 (2012).

[7] J. Gouveia, R. Z. Robinson, R. R. Thomas, Polytopes of minimum positive semidefinite rank, Discrete \& Computational Geometry 50 (3) (2013) 679-699. 